\title{
CD10 and osteopontin expression in dentigerous cyst and ameloblastoma
}

\author{
Shaimaa M Masloub, Adel M Abdel-Azim and Ehab S Abd Elhamid ${ }^{*}$
}

\begin{abstract}
Aims and Objectives: To investigate the expression of CD10 and osteopontin in dentigerous cyst and ameloblastoma and to correlate their expression with neoplastic potentiality of dentigerous cyst and local invasion and risk of local recurrence in ameloblastoma.

Methods: CD10 and osteopontin expression was studied by means of immunohistochemistry in 9 cases of dentigerous cysts (DC) and 17 cases of ameloblastoma. There were 7 unicystic ameloblastoma (UCA) and 10 multicystic ameloblastoma (MCA). Positive cases were included in the statistical analysis, carried on the tabulated data using the Open Office Spreadsheet 3.2.1 under Linux operating system. Analysis of variance and correlation studies were performed using "R" under Linux operating system (R Development Core Team (2010). Tukey post-hoc test was also performed as a pair-wise test. The significant level was set at 0.05 .

Results: High CD10 and osteopontin expression was observed in UCA and MCA, and low CD10 and osteopontin expression was observed in DC. Significant correlation was seen between CD10 and osteopontin expression and neoplastic potentiality of DC and local invasion and risk of recurrences in ameloblastoma.

Conclusions: In DC, high CD10 and osteopontin expression may indicate the neoplastic potentiality of certain areas. In UCA \& MCA, high CD10 and osteopontin expression may identify areas with locally invasive behavior and high risk of recurrence.
\end{abstract}

\section{Background}

Dentigerous cyst (DC) is the most common developmental cyst in the oral cavity, accounting for $20 \%$ of the developmental cysts of the jaws, and is almost always associated with the crown of a tooth attached to the cemento-enamel junction. It is believed to originate from the accumulation of fluid between the reduced enamel epithelium and the tooth crown, thus expanding the follicle beyond the $3 \mathrm{~mm}$ normal diameter and hence is usually associated with impacted or un-erupted teeth [1].

Ameloblastoma is an uncommon benign, locally aggressive odontogenic neoplasm that accounts for approximately $10 \%$ of all tumors that arise in the mandible and maxilla [2]. Although the etiology is unknown, ameloblastoma is believed to develop from various sources of odontogenic epithelia, including dental follicular lining epithelium [3]. Ameloblastoma is classified

\footnotetext{
* Correspondence: ihabema2003@yahoo.com

Oral Pathology Department, Faculty of Dentistry, Ain Shams University, Cairo, Egypt
}

\section{Biomed Central}

as central or peripheral. Central ameloblastomas are classified as multicystic/solid (MCA) or unicystic (UCA) [4]. Multicystic/solid ameloblastomas tend to be more aggressive and have a higher likelihood of recurrence after surgery compared with unicystic and peripheral ameloblastomas [5].

CD 10 cell surface glycoprotein was initially identified as a $100 \mathrm{KDa}$ tumor associated antigen (common acute lymphoblastic leukemia antigen, CALLA) on human acute lymphoblastic leukemias and other lymphoid malignancies with an immature phenotype [6]. CD10 is expressed on the surface of a variety of normal and neoplastic hematopoietic and lymphoid cells, including lymphoid precursor cells, germinal center B lymphocytes and some epithelial cells [7].

The specialized effects of the action of CD10/Neutral Endopeptidase (NEP) are attributable to the substrates present in different tissues, at points of contact between cells and extracellular matrix or between adjacent cells; surface CD10 would then regulate the local concentrations of specific peptides [8]. CD10 may also play an 
important role in maintenance of homeostasis, neoplastic transformation, and tumor progression [9]. Recent works suggested that CD10 expression in cancer cells could have a role both in apoptosis and proliferation [10], while CD10 expression in intratumoral stromal cells may also contribute to tumor progression [11]. High CD10 expression was associated with poor prognosis in various tumors like breast carcinoma [11], malignant melanoma [12], cutaneous basal cell and squamous cell carcinoma [13] and oral squamous cell carcinoma [14].

Meanwhile, osteopontin (OPN) was identified independently, together with bone sialoprotein (BSP), as a major sialoprotein in the extracellular matrix of bone and the 2 proteins were initially called bone sialoprotein (BSP I) and bone sialoprotein II (BSP II), respectively [15]. Constitutive expression of OPN exists in several cell types but induced expression has been detected in T lymphocytes, epidermal cells, bone cells, macrophages, and tumor cells in remodeling processes such as inflammation, ischemia-reperfusion, bone resorption, and tumor progression [16].

OPN has been shown to be multifunctional, with activities in cell migration, cell survival, inhibition of calcification, regulation of immune cell function, and control of tumor cell phenotype [17]. Binding of OPN to tumor cell membrane receptor CD44v6 can enhance tumor cell motility [18] and increase the immune adaptation of OPN-expressing cells [19]. OPN can also trigger integrin-mediated signal transduction, which, in turn, leads to osteoclast activation [20]. Ligation of OPN with integrin $\alpha 5 \beta 3$ on vascular endothelial cells induces neovascularization by up-regulating endothelial cell migration, survival, and lumen formation during angiogenesis [21].

High OPN expression was associated with poor prognosis in various tumors like prostate carcinoma [22], breast carcinoma [23] and cutaneous squamous cell carcinoma [24]. Concerning ameloblastoma, high OPN expression was reported in both unicystic and multicystic ameloblastoma [25].

The present study was conducted to investigate the expression of CD10 and osteopontin in dentigerous cyst and ameloblastoma and to correlate their expression with neoplastic potentiality of dentigerous cyst and local invasion and risk of local recurrence in ameloblastoma.

\section{Methods}

\section{Materials}

Twenty-six formalin fixed paraffin-embedded archival blocks of dentigerous cyst and ameloblastoma were obtained from the archives of the oral pathology department, Ain Shams University and National Cancer Institute, Cairo University. Data of the archival paraffin blocks included the histopathological diagnosis of each case as well as history of recurrence in cases diagnosed as multicystic ameloblastoma. Each case was then coded and patient's name was not shown for ethical reasons. Nine cases were diagnosed as dentigerous cyst, seven cases were diagnosed as unicystic ameloblastoma, and ten cases were diagnosed as multicystic ameloblastoma. To confirm the diagnosis $5 \mu \mathrm{m}$ thick sections were cut and mounted on glass slides, sections were stained with haematoxylin and eosin stain and examined by light microscope.

\section{Immunohistochemical procedures}

For all specimens $4 \mu \mathrm{m}$ sections were cut and mounted on positively charged glass slides. Sections were deparaffinized with xylene and rehydrated in graded ethyl alcohol, and sections were immersed in citrate buffer solution of $\mathrm{pH} 4.8$ and were put in the microwave oven before staining procedures. For immunostaining a universal kit (Lab Vision) was used, peroxidase anti- peroxidase method of immunostaining using the streptavidinbiotin system was carried out, and 3\% hydrogen peroxide was applied to the sections to block the endogenous peroxidase activity. Sections were immunostained using the concentrated primary monoclonal antibody (clone 56C6) against CD10 (Thermo Fisher Scientific Laboratories, Ltd, United Kingdom), and monoclonal lyophilized antibody (clone OP3N) against OPN (Visionbiosystems Novocastra ${ }^{\mathrm{TM}}$ Laboratories, Ltd, United Kingdom) and then incubated overnight at room temperature after rinsing with PBS (phosphate buffered saline) solution. Sections were then covered by the link antibody followed by the streptavidin labeling antibody; after rinsing with $\mathrm{PBS}, \mathrm{DAB}$ chromogen was applied to the sections followed by counter stain, then sections were dehydrated in graded alcohol, cleared in xylene and mounted.

\section{Assessment}

For each positive section, four microscopic fields showing highest immunopositivity were selected and photomicrographs were captured at a magnification of $20 \mathrm{X}$. This was performed using a video camera (C5060, Olympus, Japan) which was mounted on a light microscope (BX60, Olympus, Japan). Images were then transferred to the computer system for analysis. All the steps for immunohistochemical evaluation were carried out using image analysis software (Image J, 1.41a, NIH, USA).

\section{Statistical Analysis}

Positive cases were included in the statistical analysis, carried on the tabulated data using Open Office Spreadsheet 3.2.1 under Linux operating system. Analysis of 
variance and correlation studies were performed using " $R$ " under Linux operating system (R Development Core Team (2010). Analysis Of Variance (ANOVA) test was used to test the significance of mean differences within dentigerous cyst and ameloblastoma. Post Hoc test was used when ANOVA test revealed a significant difference, to assess the significance of differences within dentigerous cyst and ameloblastoma.

The results were considered significant when $P$ value $\leq 0.05$. Welch Two Sample t-test was done to compare the expression of CD10 or OPN in primary versus recurrent ameloblastoma. The statistical tests performed included Pearson's correlation analysis to test correlation between CD10 and OPN expression in dentigerous cyst and ameloblastoma.

\section{Results}

A) Immunohistochemical Results

Positive CD10 and OPN staining was found in all cases examined in the present study. The positive cases showed a homogenous and brownish immunostaining. For CD10: In DC, cytoplasmic \& membranous immunoreactivity was seen mainly in the superficial layers of the epithelial lining (Figure 1). In the intraluminal UCA, most of the central cells of epithelial strands showed membranous reaction, while few of these cells showed both membranous and cytoplasmic reaction (Figure 2). On the other hand, in the mural cases, the epithelial cells facing the cystic cavity showed both membranous and cytoplasmic reaction, while basal and suprabasal cells were immunonegative. The stellate reticulum like cells in the mural strands showed cytoplasmic reaction (Figure 3). In MCA, the neoplastic epithelial cells showed cytoplasmic and

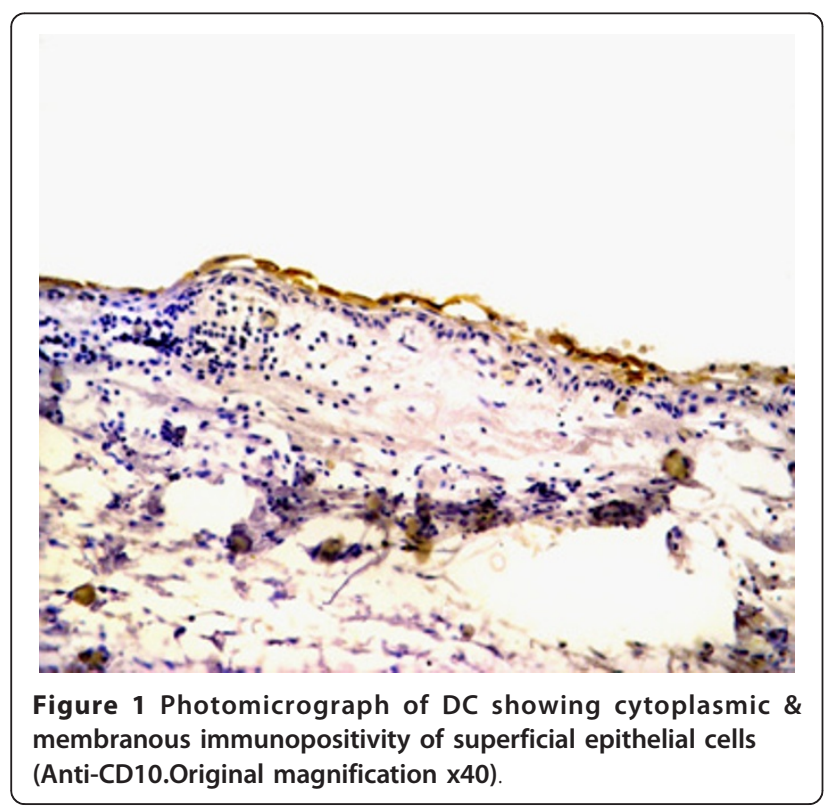

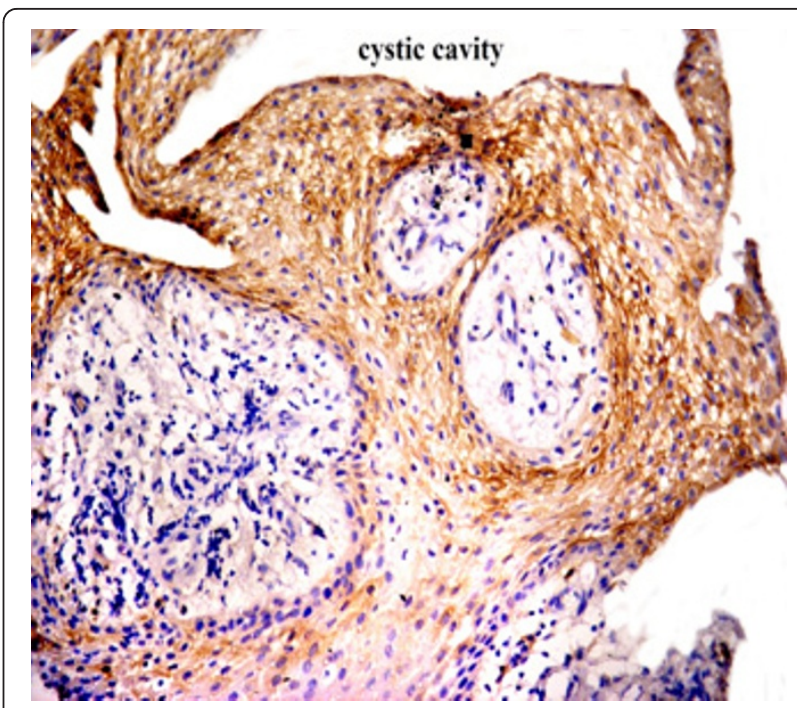

Figure 2 Photomicrograph of intraluminal UCA showing cytoplasmic \& membranous reaction of central cells of epithelial strands (red arrow) (Anti-CD10. Original magnification $\times 40$ ).

membranous immunopositivity (Figure 4). For OPN: In DC, cytoplasmic immunoreactivity was seen in epithelial cells, inflammatory cells and endothelial cells of blood vessels (Figure 5). In intraluminal UCA, the tumor cells and inflammatory cells in the stroma showed cytoplasmic reaction (Figure 6). In mural UCA, cytoplasmic immunopositivity in ameloblast like cells \& few stellate reticulum like cells was seen resulting in a peritumoral positive reaction (Figure 7). In MCA, few stellate reticulum like cells showed cytoplasmic reaction, while most of the ameloblast like cells showed cytoplasmic reaction.

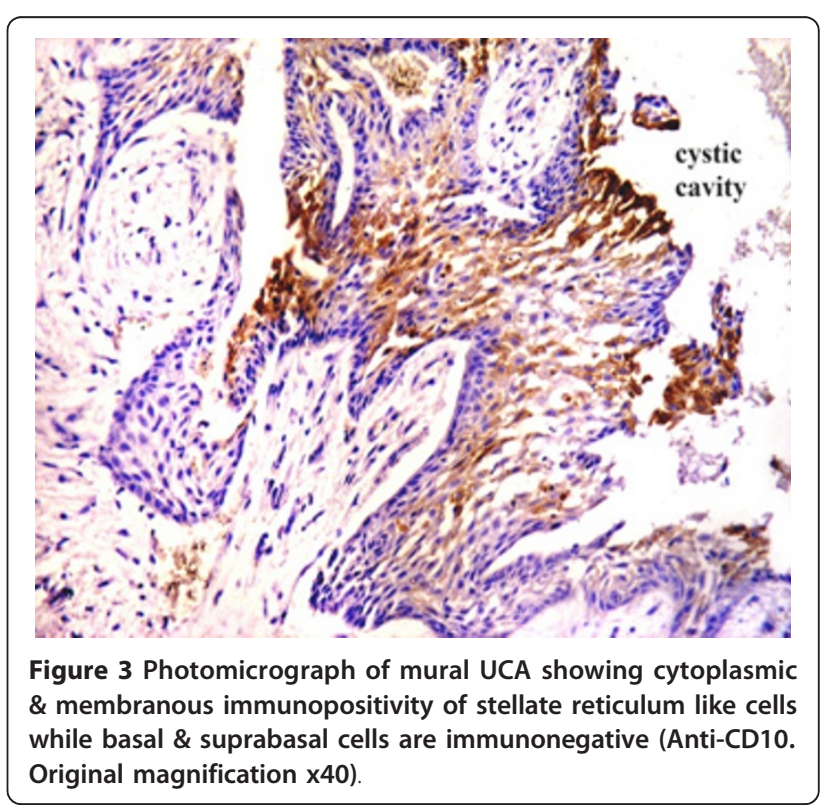




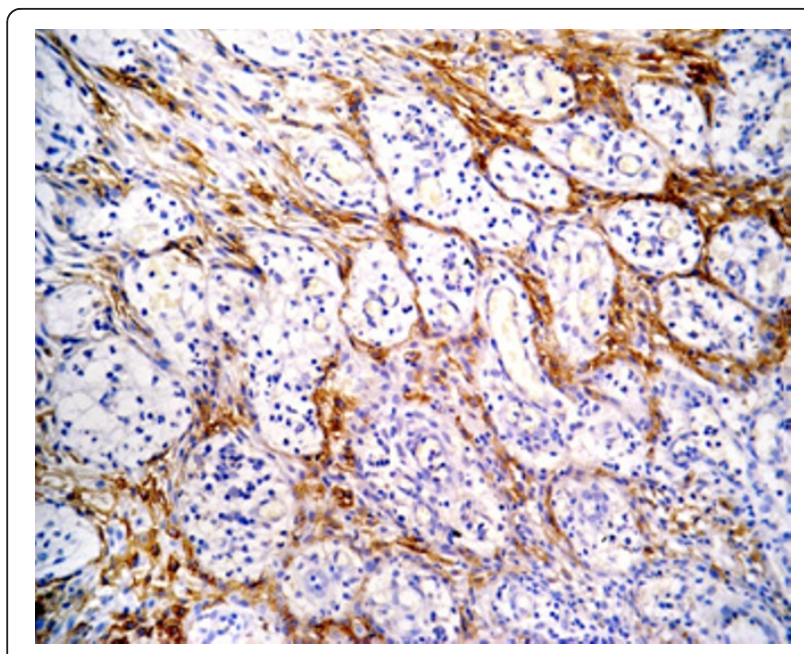

Figure 4 Photomicrograph of plexiform MCA showing cytoplasmic immunopositivty of neoplastic epithelial strands (Anti-CD10.Original magnification $\mathrm{x} 40$ ).

Peritumoral reaction was also seen in the stroma surrounding the tumor (Figure 8 ).

\section{B) Statistical Results}

Tukey pair wise test revealed a statistically significant difference in the expression of CD10 in DC \& MCA, and in UCA \& MCA (p values less than 0.001). On the other hand, there was no statistically significant difference in the expression of CD10 in UCA \& DC (Table 1).

The Tukey pair-wise test showed a statistically significant difference in the expression of OPN in MCA \& $\mathrm{DC}$, and in UCA \& DC (p values less than 0.001). On

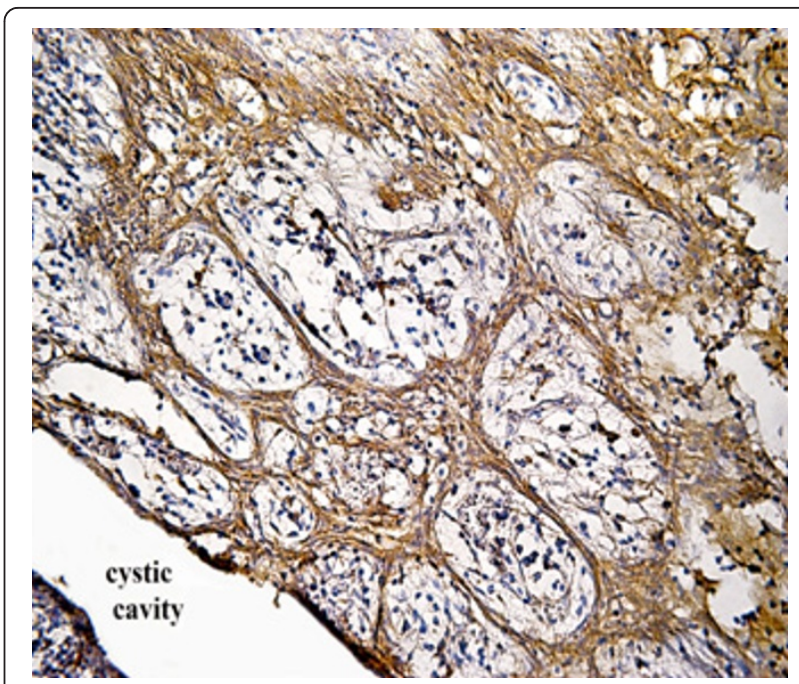

Figure 6 Photomicrograph of intraluminal plexiform variant of UCA showing cytoplasmic immunopositivity in tumor cells forming strands (Anti-OPN. Original magnification $\times 40$ ).

the other hand, there was no statistically significant difference in the expression of OPN in UCA \& MCA ( $p$ values more than 0.001 ) (Table 2). Statistical results of Welch Two Sample t-test revealed a statistically insignificant difference between CD10 or OPN mean area fraction in recurrent versus primary MCA (Tables 3 and 4). Pearson's correlation test showed a significant strong direct positive correlation between CD10 and OPN immunopositivity in either dentigerous cyst or ameloblastoma ( $\mathrm{r}$ value $=0.622$, P-value $<0,001$ ) (Figure 9).
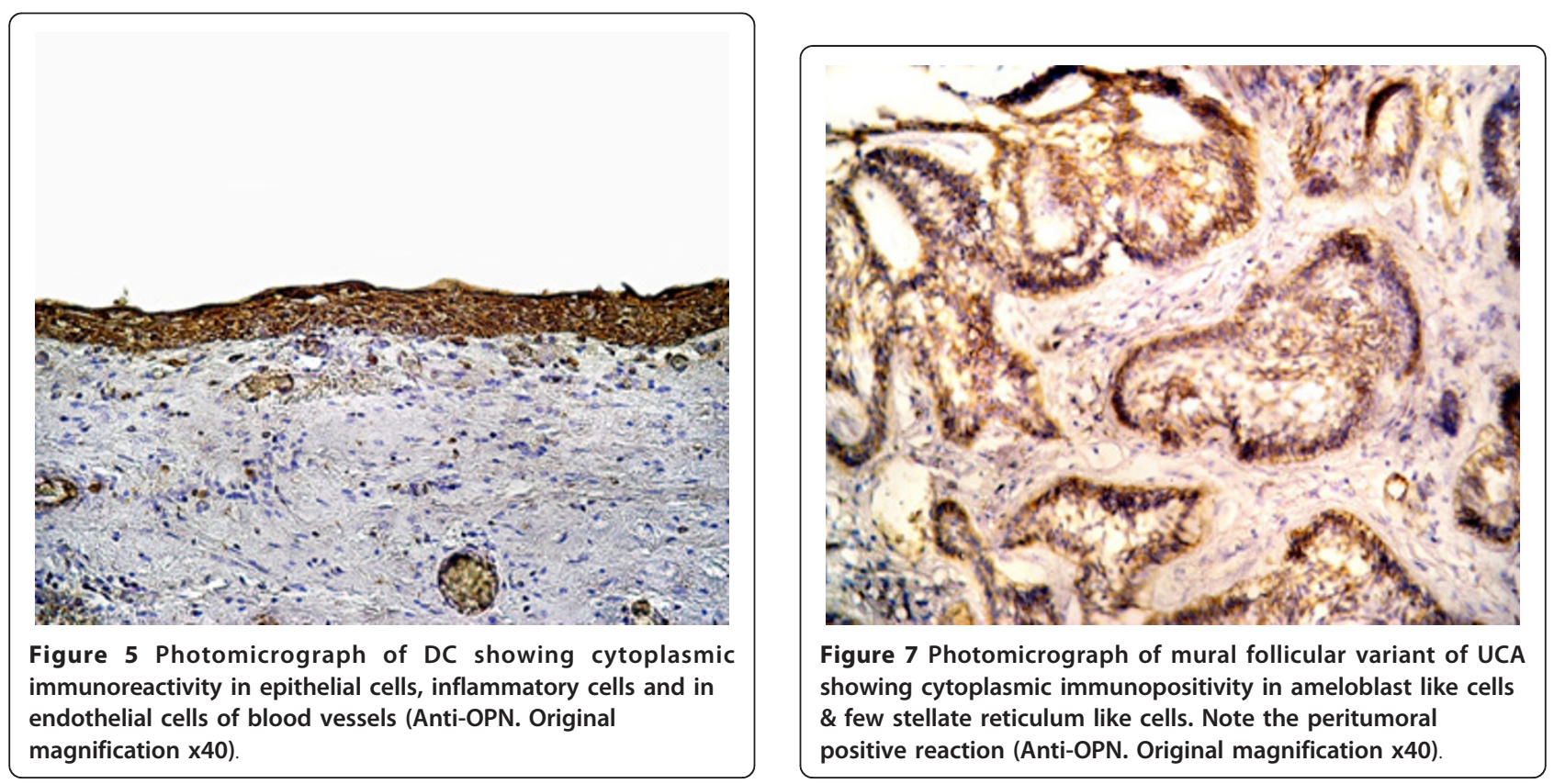


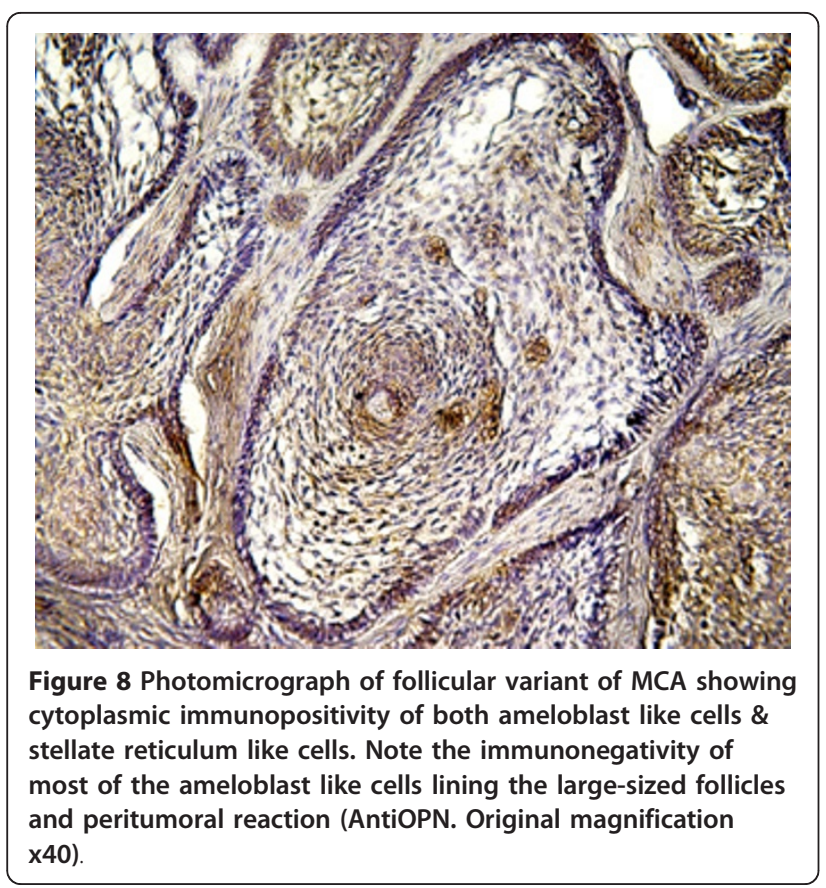

\section{Discussion}

Dentigerous cyst (DC) is the most common developmental cyst in the oral cavity, accounting for $20 \%$ of the developmental cysts of the jaws, and is almost always associated with the crown of a tooth attached to the cemento-enamel junction [1]. Ameloblastoma is an uncommon benign, locally aggressive odontogenic neoplasm that accounts for approximately $10 \%$ of all tumors that arise in the mandible and maxilla [2]. Ameloblastoma is classified as central or peripheral. Central ameloblastomas are classified as multicystic/solid (MCA) or unicystic (UCA) [4].

The immunohistochemical results of the present study showed that cytoplasmic \& membranous CD10 immunoreactivity was seen mainly in the superficial layers of the epithelial lining of DC. These results were found to be in accordance to that reported by Liapatas et al [26] who reported CD10 expression in periapical granulomas

Table 1 Descriptive statistics (CD10)

\begin{tabular}{|c|c|c|c|c|}
\hline Lesions & \multicolumn{2}{|c|}{ Mean } & Std.Deviation & Std.Error \\
\hline DC & \multicolumn{2}{|c|}{3.033} & 2.343 & 36 \\
\hline MCA & \multicolumn{2}{|c|}{9.995} & 4.915 & 40 \\
\hline UCA & \multicolumn{2}{|c|}{4.925} & 2.851 & 28 \\
\hline \multicolumn{5}{|c|}{ Tukey pair-wise test for CD10. } \\
\hline Lesion & diff & Lower & upper & $\mathrm{p}$-value \\
\hline MCA-DC & 6.962 & 4.838 & 9.085 & 0.000 \\
\hline UCA-DC & 1.892 & -0.438 & 4.21 & 0.154 \\
\hline UCA-MCA & -5.070 & -7.348 & -2.792 & 0.000 \\
\hline
\end{tabular}

* The mean difference is significant at the 0.05 level
Table 2 Descriptive statistics (OPN)

\begin{tabular}{|c|c|c|c|c|}
\hline lesions & \multicolumn{2}{|c|}{ Mean } & Std.Deviation & Std.Error \\
\hline DC & \multicolumn{2}{|c|}{8.9} & 3.3 & 36 \\
\hline MCA & \multicolumn{2}{|c|}{18.2} & 7.1 & 40 \\
\hline UCA & \multicolumn{2}{|c|}{17.0} & 3.8 & 28 \\
\hline \multicolumn{5}{|c|}{ Tukey pair-wise test for OPN } \\
\hline lesion & diff & lower & upper & P-value \\
\hline MCA-DC & 9.31 & 6.29 & 12.33 & 0.000 \\
\hline UCA-DC & 8.14 & 4.83 & 11.45 & 0.000 \\
\hline UCA-MCA & -1.17 & -4.41 & 2.07 & 0.781 \\
\hline
\end{tabular}

and cysts. In UCA, the results of the present study revealed that the immunohistochemical expression of CD10 in the mural cases was different from that noted in the intraluminal cases. In the intraluminal cases, CD10 was expressed in the stellate reticulum like cells and this expression was mainly membranous. On the other hand in mural cases, the epithelial cells facing the cystic cavity showed membranous and cytoplasmic immunoreactivity of CD10, while the stellate reticulum like cells in the mural strands showed mainly a cytoplasmic reaction. These results were found to be consistent with the results of other studies such as that of Granados et al. [27] and Aiad and Hanout [13] where the tumor cells in these studies showed mainly cytoplasmic expression of CD10. This difference in the expression of CD10 in the intraluminal and mural variants of UCA might explain the variable behavior of these two variants. This finding was in agreement with the argument of Rosenstein et al [28] who suggested that the luminal and intraluminal variants of UCA were non aggressive and could be treated by enucleation whereas the mural variant should be treated more aggressively and concluded that since most of the recurrent cases of ameloblastoma were of the mural type, connective tissue invasion can be considered an important microscopic sign. Linear with these data was that reported by Gardner [29] who stated that once mural invasion occurred, the UCA at this stage would act as classic intraosseous ameloblastoma and has to be treated as such. In MCA, the neoplastic epithelial cells showed cytoplasmic and membranous immunopositivity. This might be explained by the argument of Ogawa et al [30] who stated that CD10 was associated with the differentiation and growth of neoplastic cells.

Statistical analysis of the present study revealed that the CD10 mean area fraction of immunopositivity increased from DC to UCA and MCA. This could be explained by the different biological properties of CD10 which could facilitate the neoplastic transformation of DC and the locally invasive behavior of ameloblastoma. The highly aggressive and locally invasive behavior of 
Table 3 Welch Two Sample t-test (CD10)

\begin{tabular}{|c|c|c|c|c|c|c|}
\hline \multirow[t]{2}{*}{ Dependent Variable } & \multirow[t]{2}{*}{ MCA } & \multirow[t]{2}{*}{ Mean AF } & \multicolumn{2}{|c|}{ 95\% Confidence Interval } & \multirow[t]{2}{*}{ df } & \multirow[t]{2}{*}{$\mathrm{P}$ valu } \\
\hline & & & Lower Bound & Upper Bound & & \\
\hline \multirow[t]{2}{*}{ CD10 Mean Area Fraction } & Primary MCA & 4.6536 & 2.1695 & 3.3140 & 39 & 0.8178 \\
\hline & Recurrent MCA & 4.2000 & & & & \\
\hline
\end{tabular}

* The mean difference is significant at the 0.05 level

MCA could explain the presence of a statistically significant difference in CD10 expression when comparing MCA with DC and UCA as the CD10 could facilitate this aggressive behavior.

These results were consistent with the results of Iezzi et al [31] who showed that the mean area fraction of stromal CD10 immunopositivity of MCA was higher than that of UCA and peripheral ameloblastoma. Although the mean area fraction of CD10 in UCA was higher than that of $\mathrm{DC}$, this difference was found to be statistically insignificant.

The immunohistochemical results of the present study showed that OPN immunoreactivity was seen in both epithelium of DC together with underlying connective tissue. However, these results were not consistent with that reported by Wang and Liu [20] who found OPN immunopostivity in odontogenic keratocysts but not in DC. This OPN expression pattern in the epithelial lining of DC might be an early indicator of neoplastic transformation of DC into UCA. This is supported by the fact the induced expression of OPN has been detected in epidermal cells in remodeling processes as the tumor progresses [16]. Chang et al [24] further supported this argument and stated that OPN expression in both benign and malignant tumors suggested its association with the process of tumorgenesis.

The immunohistochemical results of the present study also revealed a different distribution pattern of OPN among different variants of UCA. In the intraluminal cases, OPN immunoreactivity was noted in neoplastic epithelial cells with no peritumoral stromal reaction. This expression pattern was similar to that noted in DC. This finding might explain the favorable behavior of these two subtypes as being non aggressive variants of UCA [28]. In the mural cases, the OPN expression was noted in neoplastic epithelial cells as well as peritumoral stromal tissue. This difference in OPN localization in this particular variant when compared to luminal and intraluminal UCA might explain the difference in the biological behavior of these variants. This explanation is supported by the argument of Wang and Liu [25] who stated that the tumor cell produced OPN could facilitate the tumor cell adhesion and migration in the bone resulting in tumor invasion and spread. So this weak expression of OPN in luminal and intraluminal subtypes, when compared to the mural subtype, might explain why these two subtypes have a favorable prognosis and less invasive behavior than that of the mural subtype.

In MCA, the results of the present study revealed that in both follicular and plexiform ameloblastoma, cytoplasmic OPN immunoreactivity was observed in ameloblast like cells, with cytoplasmic localization in few stellate reticulum like cells. Peritumoral reaction was also noted in the stroma surrounding the tumor. This distribution pattern was found to be consistent with that reported by Wang and Liu [25].This expression pattern of OPN could be explained by the argument of Wang and Liu [25] who stated that OPN protein is probably synthesized and secreted by stellate reticulum like cells, picked up by ameloblast like cells and released into the peritumor nest stromal tissue (transcytosis in ameloblast like cells) in ameloblastoma. They also added the possibility that a small amount of OPN is produced by ameloblast like cells also could not be ruled out [25].

Since OPN can enhance tumor cell migration, invasion and spread, activate osteoclasts, and protect cells from immune mediated cytotoxicity, the elevated expression of OPN in ameloblastoma tumor cells and peritumor nest connective tissue of ameloblastoma, can, at least, partially explain why MCA have the locally invasive behavior and high osteolytic ability [25]. It was reported that OPN expression was associated with recurrence in prostate cancer [22]. Also, odontogenic keratocyst, which is known for its high rate of recurrence, showed strong OPN immunostaining in both its

Table 4 Welch Two Sample t-test (OPN)

\begin{tabular}{|c|c|c|c|c|c|c|}
\hline \multirow[t]{2}{*}{ Dependent Variable } & \multirow[t]{2}{*}{ MCA } & \multirow[t]{2}{*}{ Mean AF } & \multicolumn{2}{|c|}{ 95\% Confidence Interval } & \multirow[t]{2}{*}{ df } & \multirow[t]{2}{*}{$P$ value } \\
\hline & & & Lower Bound & Upper Bound & & \\
\hline \multirow[t]{2}{*}{ OPN Mean Area Fraction } & Primary MCA & 12.4393 & 3.6277 & 5.5415 & 39 & 0.2741 \\
\hline & Recurrent MCA & 16.0667 & & & & \\
\hline
\end{tabular}

* The mean difference is significant at the 0.05 level 


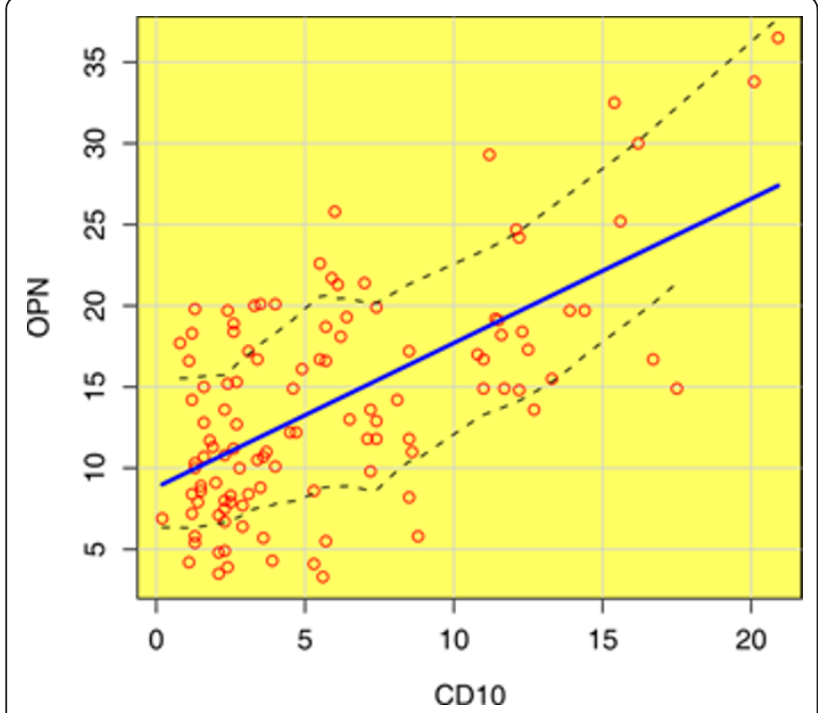

Figure 9 Pearson's correlation of CD10 with OPN showing the trend line and least square lines (dotted).

epithelial lining and underlying connective tissue [20]. So, the elevated expression of OPN in ameloblastoma might explain the high recurrence rate of this lesion.

In different lesions included in the present study, OPN expression in inflammatory cells could be explained by the fact induced expression has been detected in T lymphocytes, macrophages, and in remodeling processes such as inflammation, ischemia-reperfusion, bone resorption, and tumor progression [16]. Also, detected OPN expression along the walls of blood vessels might indicate the possible role of OPN in neovascularization. This could be explained by the binding properties of OPN, where the ligation of OPN with integrin $\alpha 3 \beta 5$ on vascular endothelial cells induces neovascularization by upregulating endothelial cell migration, survival and lumen formation during angiogenesis. This might explain the OPN immunoreactivity in endothelial cells noted in the present study $[16,21]$. These angiogenic properties of OPN would increase the rate of tumor growth. The statistical analysis of the present study revealed that the mean area fraction of OPN immunopositivity of UCA and MCA was higher than that of DC. The difference in the mean area fraction of OPN in UCA (mural variant) and MCA when compared to DC might explain the locally invasive behavior of these two variants. This difference was found to be statistically significant. In UCA \& MCA, the statistically insignificant difference of OPN expression in these two groups could be due to that most of the UCA studied were of the mural type, as it was reported that the behavior of the mural type is similar to that of the classic intraosseous MCA [28]. Statistical results of the present study revealed a statistically insignificant difference of either
CD10 or OPN mean area fraction when comparing recurrent versus non recurrent MCA. This might be due to inconsistent data gathered from a small sample size of the present study. Thus, further studies utilizing more recurrent cases of MCA are highly recommended.

A Pearson's correlation study showed that there was a strong significant positive correlation between the levels of $\mathrm{CD} 10$ and OPN expression $(\mathrm{R}$ value $=0.622)$. This correlation was proven to be statistically significant (P value $<0.001)$. This could be explained by the different biological properties of CD10 regarding neoplastic transformation and tumor progression [9]. OPN enhancement of tumor cell migration, invasion and spread [32], activation of osteoclast [20], and protection of cells from immune mediated cytoxicity [19] might also add to understand this positive association between the two markers. So these two markers could function in a synergistic way to facilitate the neoplastic transformation of DC and the locally invasive behavior of ameloblastoma.

\section{Conclusions}

Based upon the results of the present study, it could be concluded that, in DC, high CD10 and osteopontin expression might predict the neoplastic potentiality of the epithelial lining of this cyst. Also, in UCA \& MCA, high $\mathrm{CD} 10$ and osteopontin expression might be a useful tool to identify areas with locally invasive behavior and high risk of recurrence.

\section{Authors' contributions}

SMM participated in the study design, collection of the background references, performing the immunohistochemical technique and photomicrography of the results, writing the discussion of the results and participated in the collection of the background references.

AMA participated in the study design, revised the results and discussion, carried out the statistical analysis.

ESA participated in the study design, revised the image analysis of immunohistochemical results, revised the discussion of the results, participated in the collection of the background references, carried out the sequence alignment, and drafted the manuscript.

All authors read and approved the final manuscript.

\section{Competing interests}

The authors declare that they have no competing interests.

Received: 14 April 2011 Accepted: 24 May 2011 Published: 24 May 2011

\section{References}

1. Shibata Y, Asaumi J, Yanagi Y, Kawai N, Hisatomi M, Matsuzaki H, Konouchi $H$, Nagatsuka H, Kishi K: Radiographic examination of dentigerous cysts in the transitional dentition. Dentomaxillofac Radiol 2004, 33:17-20.

2. Zemann W, Feichtinger $M$, Kowatsch $E$, Kärcher H: Extensive ameloblastoma of the jaws: surgical management and immediate reconstruction using microvascular flaps. Oral Surg Oral Med Oral Pathol Oral Radiol Endod 2007, 103:190-196.

3. Kumamoto $\mathrm{H}$, Ooya $\mathrm{K}$ : Immunohistochemical detection of retinoblastoma protein and E2 promoter-binding factor-1 in ameloblastomas. J Oral Pathol Med 2006, 35:183-189. 
4. Carlson E, Marx R: The ameloblastoma: primary, curative surgical management. J Oral Maxillofac Surg 2006, 64:484-494.

5. Lau S, Samman N: Recurrence related to treatment modalities of unicystic ameloblastoma: a systematic review. Int J Oral Maxillofac Surg 2006, 35:681-690.

6. Greaves M, Hairi G, Newman R, Sutherland D, Ritter M, Ritz J: Selective expression of the common acute lymphoblastic leukemia (gp100) antigen on immature lymphoid cells and their malignant counterparts. Blood 1983, 61:628-639.

7. Xu Y, McKenna R, Kroft S: Comparison of multiparameter flow cytometry with cluster analysis and immunohistochemistry for the detection of CD10 in diffuse large B-cell lymphomas. Mod Pathol 2002, 15:413-419.

8. Letarte M, Ishii E: The potential role of CD10 / neutral endopeptidase 24.11 in the immune system. Advances in neuroimmunology 1993, 3:183-194

9. Papandreou C, Usmani B, Geng Y, Bogenrieder T, Freeman R, Wilk S, Finstad C, Reuter V, Powell C, Scheinberg D, Magill C, Scher H, Albino A, Nanus D: Neutral endopeptidase 24.11 loss in metastatic human prostate cancer contributes to androgen-independent progression. Nat Med 1998, 4:50-57

10. Suzuki T, Ino K, Kikkawa F, Uehara C, Kajiyama H, Shibata K, Mizutani S: Neutral endopeptidase/CD10 expression during phorbol ester-induced differentiation of choriocarcinoma cells through the protein kinase Cand extracellular signal-regulated kinase-dependent signaling pathway. Placenta 2002, 23:475-482.

11. Iwaya K, Ogawa H, Izumi M, Kuroda M, Mukai K: Stromal expression of CD10 in invasive breast carcinoma: a new predictor of clinical outcome. Virchows Arch 2002, 440:589-593.

12. Bilalovic N, Sandstad B, Golouh R, Ja Nesland J, Selak I, Torlakovic E: CD10 protein expression in tumor and stromal cells of malignant melanoma is associated with tumor progression. Modern Pathology 2004, 17:1251-1258.

13. Aiad $\mathrm{H}$, Hanout $\mathrm{H}$ : Immunohistochemical Expression of CD10 in Cutaneous Basal and Squamous Cell Carcinomas. Journal of the Egyptian Nat. Cancer Inst 2007, 19:195-201.

14. Piattelli A, Fioroni M, lezzi G, Perrotti V, Stellini E, Piattelli M, Rubini C: CD10 expression in stromal cells of oral cavity squamous cell carcinoma: a clinic and pathologic correlation. Oral Diseases 2006, 12:301-304.

15. Zhang O, Domenicucci C, Goldberg H, Wrana I, Sodek J: Characterization of fetal porcine bone sialoproteins: secreted phosphoprotein I (SPPI, osteopontin), bone sialoprotein, and a $23 \mathrm{kDa}$ glycoprotein. Demonstration that the $23 \mathrm{kDa}$ glycoprotein is derived from the carboxy-terminus of SPPI. Biol Chem 1990, 265:7583-7589.

16. Weber G: The metastasis gene osteopontin: a candidate target for cancer therapy. Biochim. Biophys. Acta 2001, 1552-61.

17. Weber G, Cantor H: Cytokine Growth Factor. Rev 1996, 7:241-248.

18. Lee J, Wang M, Sudhir P, Chen G, Chi C, Chen J: Osteopontin promotes integrin activation through outside-in and inside-out mechanisms: OPNCD44V interaction enhances survival in gastrointestinal cancer cells. Cancer Res 2007, 67:2089-97.

19. Jain A, Karadag A, Fohr B, Fischer L, Fedarko N: Three SIBLINGs (small integrin-binding ligand, N-linked glycoproteins) enhance factor $\mathrm{H}^{\prime} \mathrm{s}$ cofactor activity enabling MCP-like cellular evasion of complementmediated attack. J Biol Chem 2002, 277:13700-8.

20. Wang Y, Liu B: High Expression of Osteopontin and CD44v6 in Odontogenic Keratocysts. J Formos Med Assoc 2009, 108:286-292.

21. Bayless K, Salazar R, Davis G: RGD-dependent vacuolation and lumen formation observed during endothelial cell morphogenesis in threedimensional fibrin matrices involves the alpha (v)beta(3) and alpha(5) beta(1) integrins. Am J Pathol 2000, 156:1673.

22. Caruso D, Carmack A, Lokeshwar V, Duncan R, Soloway M, Lokeshwar B: Osteopontin and Interleukin-8 Expression is independently associated with Prostate Cancer Recurrence. Clin Cancer Res 2008, 14:4111-4118.

23. Urquidi V, Kawai K, Agarwal D, Woodman A, Tarin D, Goodison S: Contrasting expression of thrombospondin-1 and osteopontin correlates with absence or presence of metastatic phenotype in an isogenic model of spontaneous human breast cancer metastasis. Clin Cancer Res 2002, 8:61-74.

24. Chang P, Harkins L, Hsieh Y, Hicks P, Sappayatosok K, Yodsanga S, Swasdison S, Chambers A, Elmets C, Ho K: Osteopontin Expression in Normal Skin and Non-melanoma Skin Tumors. Journal of Histochemistry \& Cytochemistry. 2008, 56:57-66.
25. Wang Y, Liu B: Expression of osteopontin and its receptors in ameloblastomas. Oral Oncol 2008, 7:1-5

26. Liapatas S, Nakou M, Rontogianni D: Inflammatory infiltrate of chronic periradicular lesions: an immunohistochemical study. International Endodontic Journal 2003, 36:464-471.

27. Granados R, Carrillo R, Na'jera L, Villanueva M, Patro'n M: Psammomatoid ossifying fibromas: Immunohistochemical analysis and differential diagnosis with psammomatous meningiomas of craniofacial bones. Oral Surg Oral Med Oral Pathol Oral Radiol Endod 2006, 101:614-9.

28. Rosenstein T, Pogrel M, Smith R, Regezi J: Cystic ameloblastoma-Behaviour and treatment of 21 cases. J Oral Maxillofac Surg 2001, 59:1311-16.

29. Gardner D: Some current concepts on the pathology of ameloblastoma. Oral and maxillofacial pathology 1996, 82(6):660-662.

30. Ogawa H, Iwaya K, Izumi M, Kuroda M, Serizawa H, Koyanagi Y, Mukai K. Expression of $\mathrm{CD} 10$ by stromal cells during colorectal tumor development. Hum Pathol 2002, 33:806-811.

31. lezzi G, Piattelli A, Rubini C, Artese L, Goteri G, Fioroni M, Carinci F: CD10 expression in stromal cells of ameloblastoma variants. Oral Surg Oral Med Oral Pathol Oral Radiol Endod 2008, 105:206-9.

32. Furger K, Allan A, Wilson S, Hota C, Vantyghem S, Postenka C, Al-Katib W, Chambers A, Tuck A: Beta(3) integrin expression increases breast carcinoma cell responsiveness to the malignancy-enhancing effects of osteopontin. Mol Cancer Res 2003, 1:810.

doi:10.1186/1746-1596-6-44

Cite this article as: Masloub et al.: CD10 and osteopontin expression in dentigerous cyst and ameloblastoma. Diagnostic Pathology 2011 6:44.

\section{Submit your next manuscript to BioMed Central and take full advantage of:}

- Convenient online submission

- Thorough peer review

- No space constraints or color figure charges

- Immediate publication on acceptance

- Inclusion in PubMed, CAS, Scopus and Google Scholar

- Research which is freely available for redistribution

Submit your manuscript at www.biomedcentral.com/submit

C) Biomed Central 